\title{
Aufzucht, Spermaproduktion und Befruchtungserfolg bei Ayrshirebullen in der Besamungsstation Salpausselkä
}

\author{
G. KLAUtscheK \\ Sektion Tierproduktion, Universität Rostock, DDR \\ U. Uusisalmi \\ Institut für Tierzüchtung, Universität Helsinki, Finnland
}

Eingegangen am 26.1.1973

\section{Results of performance testing and semen production, and non-return percentages of Ayrshire bulls in the Salpausselkä A. I. Society}

\author{
G. KlautscheK \\ Sektion Tierproduktion, Universität Rostock, DDR \\ U. Uusisalmi \\ Helsinki University, Department of Animal Breeding
}

\begin{abstract}
The rearing, feeding and management of A. I. bulls are of importance to their semen production and fertility. Analyses were consequently made of performance testing results, quantity and quality of semen production and insemination results in respect of 44 Ayrshire bulls. Non-return rates were calculated from a total of 57289 first inseminations.

The Ayrshire bulls were reared to the age of one year at the Humppila Performance Testing Station in 1967-69. The bulls were weighed during the experiment at intervals of 30 days. Measurements were taken at the age of 180 days and 365 days. The quantity and quality of semen production of the bulls were measured from the age of 12 months to the age of 24 months. The non-return rates were calculated for at least the first two years of insemination. The number of first inseminations varied from bull to bull between 666 and 3509 . Non-return rates for the bulls varied from $62.3 \%$ to $90.0 \%$ in the first year of insemination and from $58.3 \%$ to $89.2 \%$ in the second. The differences between the bulls were significant.

In the study Uusisalmi et al. (1973) found that bulls that had gained well also produced semen well. In the present study by contrast, the results of the performance testing showed a negative correlation with the non-return rate. The non-return rates likewise showed a negative correlation with the measurements taken at the age of 180 days and 365 days. When the bulls were classified as light and heavy, the non-return percentages obtained with the lightweight bulls were found to be significantly higher than those for the heavy bulls.
\end{abstract}


The correlations were calculated between the non-return rates of the bulls on the one hand and the absolute and relative milk and fat yields of their parents on the other, and the correlations were generally found to be negative. However, only the correlation between the non-return rate of the bulls and the fat percentage of the sires was statistically significant $\left(\mathrm{r}=-0.45^{* *}\right)$. The results may be regarded as suggesting that the increasing of production level through breeding might lead to reduced non-return rates.

The study shows that the semen production capacity and the NR \% of A. I. bulls should be assessed together with the characteristics of milk and meat yield, in order to prevent in due time any unfavourable development in fertility characteristics.

Die zentrale Stellung der Vatertiere in der Rinderzucht macht es notwendig, allseitige Kenntnisse über die Leistungen der Bullen zu erlangen. Neben dem Wachstum und dem Futterverzehr muss der Zuchtwert für die ökonomisch relevanten Merkmale wie Milch- und Fleischleistung sowie die Reproduktionsfähigkeit der Tiere festgestellt werden. In Finnland werden die Bullen in zentralen Stationen aufgezogen und geprüft. Anschliessend werden die Bullen auf die einzelnen Besamungsstationen gestellt und ihr Zuchtwert wird ermittelt.

Das Bemühen der Züchter ist während des gesamten Lebens der Zuchtbullen darauf gerichtet, durch hervorragende Umweltgestaltung vorzügliche Leistungseigenschaften bei den Tieren zu entwickeln. Zwischen Aufzucht, Spermaproduktion und Fruchtbarkeit bestehen allgemein bekannte Zusammenhänge. Ziel dieser Untersuchungen ist es, die Wechselverhältnisse zwischen der Aufzucht der Bullen und deren Spermaproduktion sowie den Besamungsergebnissen zu studieren, um einen Beitrag zur Verbesserung der Umweltgestaltung für die Zuchtbullen zu leisten.

\section{Material und Methode}

Untersucht wurden 44 finnische Ayrshirebullen, die in den Jahren 196769 in der Eigenleistungsprüfstation Humppilan kasvatusasema aufgezogen wurden. Mit 60 Tagen beginnend, wurden die Tiere während der Aufzucht alle 30 Tage gewogen. Zusätzlich wurden bei den Jungbullen am 180. Tag und am 365. Tag der Brustumfang, die Körperlänge, die Widerristhöhe, die Kreuzbeinhöhe, die ,Beckenlänge, die Beckenbreite und die Brustbreite gemessen. Die Fütterung entsprach den Forderungen des Zuchtverbandes und war für alle Tiere gleichmässig. Die Auswertung der Spermaprotuktion erfolgte von den in der Besamungsstation Salpausselkä erfassten Merkmalen:

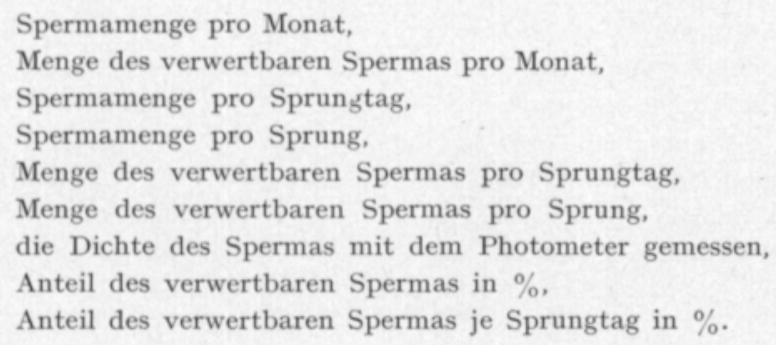

Die Spermaproduktion wurde vom 13. bis zum 24. Lebensmonat registriert. Nur Bullen, von denen mindestens 7 Monate Samen gesammelt wurde, fanden 
bei den Berechnungen Berücksichtigung. Die Befruchtungsfähigkeit der Bullen wurde durch die Besamungsergebnisse deutlich. Von 57289 Erstbesamungen konnte die non-return Rate ausgewertet werden. Eine mathematisch-statistische Bearbeitung der Beobachtungswerte erfolgte mit Hilfe von Korrelationsanalysen, Faktorenanalysen sowie t-Tests und $\chi^{2}$-tests. Die Berechnungen wurden mit dem Routineprogramm (HyLPS 1972) des Universitätsrechenzentrums der Universität Helsinki ausgeführt.

\section{Ergebnisse}

Die 44 Ayrshirebullen wogen mit 12 Monaten $447 \mathrm{~kg}$ und nahmen täglich $1234 \mathrm{~g} \mathrm{zu}$. Ausführlich wurde die Körperentwicklung und die Spermaproduktion der untersuchten Bullen bereits bei Uusisalmi u.a. (1972) beschrieben. In dieser Arbeit sollen besonders die Zusammenhänge zur Befruchtungsfähigkeit dargestellt werden. Von jedem Bullen konnte mindestens das 1. und 2. Besamungsjahr ausgewertet werden, von einigen auch weitere Besamungsjahre. Im 1. Besamungsjahr schwankte die Zahl der Erstbesamungen von Bulle zu Bulle zwischen 20 und 1583. Im Verlaufe aller ausgewerteten Jahre waren von jedem Bullen mindestens 666 und höchstens 3509 Erstbesamungen gemacht worden. Der Anteil der erfolgreichen Erstbesamungen unterscheidet sich nicht gesichert zwischen den Jahren. Mittelwert und Standardabweichung, in Tabelle 1 aufgeführt, dienen zur Information

Tabelle 1. Besamungsergebnisse der untersuchten Bullen und Zuchtwerte der Väter.

\begin{tabular}{|c|c|c|}
\hline Merkmal & $\begin{array}{l}\text { Mittelwert } \\
\qquad \bar{x}\end{array}$ & $\begin{array}{c}\text { Standardab- } \\
\text { weichung } \\
\text { s }\end{array}$ \\
\hline \multicolumn{3}{|l|}{ 1. Besamungsjahr } \\
\hline 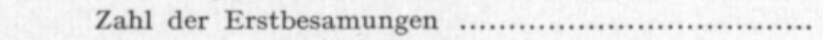 & 723 & 437.2 \\
\hline Zahl der nicht nachbesamten Tiere ........................ & 538 & 330.4 \\
\hline 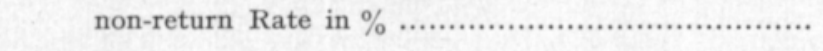 & 74.4 & 5.67 \\
\hline \multicolumn{3}{|l|}{ 2. Besamungsjahr } \\
\hline 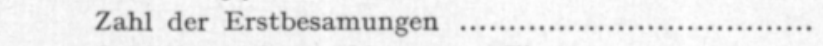 & 438 & 459.7 \\
\hline Zahl der nicht nachbesamten Tiere ....................... & 334 & 362.0 \\
\hline 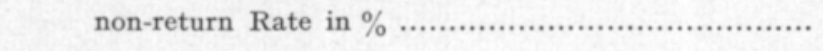 & 73.9 & 7.50 \\
\hline \multicolumn{3}{|l|}{ Alle ausgewerten Besamungsjahre (1.-4. Jahr) } \\
\hline Zahl der Erstbesamungen ...................... & 1302 & 646.8 \\
\hline 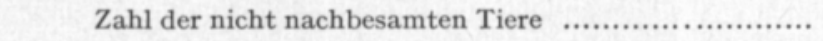 & 980 & 515.3 \\
\hline 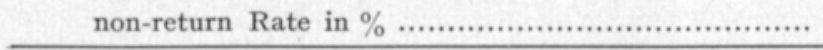 & 74.4 & 4.59 \\
\hline \multicolumn{3}{|l|}{ Zuchtwerte der Väter } \\
\hline 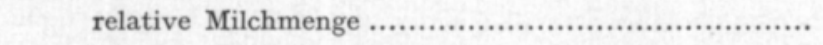 & 5.68 & 1.63 \\
\hline 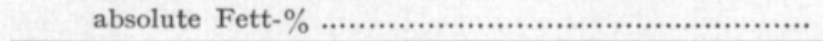 & 4.61 & 0.12 \\
\hline
\end{tabular}


über die Erstbesamungen und die non-return Ergebnisse im Untersuchungsmaterial. Im 2. Besamungsjahr wurden die Bullen bereits als "Wartebullen" behandelt und der Spermaeinsatz erfolgt unter anderen Gesichtspunkten als im 1. Jahr. Das muss berücksichtigt werden bei der Bewertung der Ergebnisse für das 1. Jahr.

Der Befruchtungserfolg für die einzelnen Bullen war recht unterschiedlich. Im 1. Besamungsjahr konnte eine non-return Rate von $62,3 \%$ bis $90,0 \%$ ermittelt werden, im 2. Jahr von $\mathbf{5 8 , 3} \%$ bis $89,2 \%$ und bei der Auswertung aller Jahre von $62,3 \%$ bis $85,3 \%$. Mit Hilfe des $\mathrm{X}^{2}$-Testes konnte ein hoch signifikanter Unterschied $(\alpha=1 \%)$ für die non-return Zahlen der Väter festgestellt werden.

Auf die Befruchtungsfähigkeit des Spermas zeigen die erzeugte Spermamenge je Bulle und die Absamhäufigkeit einen deutlichen Einfluss. Je mehr Sperma monatlich von einem Bullen gewonnen wird, um so mehr verwertbares Sperma wird er erzeugen. Die Korrelation betrug in diesem Versuch $0,72^{* * *}$. Demgegenüber verringert sich aber die Spermaqualität bei hoher Belastung der Tiere. Der Anteil des verwertbaren Spermas sinkt bei grösserer Absamhäufigkeit $r=-0,01$. Deutlich spiegelt sich dies in der Beziehung Absamungen je Sprungtag zu Anteil verwertbaren Spermas je Sprungtag wider. Die Korrelation ist $-0,35 *$. Es wurden ein, zwei oder drei Sprünge je Tag beobachtet.

Der Einfluss der Entwicklung der Bullen auf ihre Spermaproduktion und ihre Fruchtbarkeit kann durch Korrelationsrechnungen zwischen entsprechenden Beobachtungsmerkmalen in Zahlen gefasst werden. Obwohl die Beobachtungen durch lange Zeiträume getrennt sind und vielen Umwelteinflüssen unterliegen, zeigen sie doch die wesentlichsten Wechselbeziehungen deutlich auf. Für die Aufzucht, die Spermaproduktion und den Befruchtungserfolg konnten mehrere gesicherte Zusammenhänge ermittelt werden. Die Tabelle 2 gibt die wichtigsten Korrelationen für die registrierten Merkmale an. Zur besseren Auswertung der umfangreichen Korrelationsmatrix wurde eine Faktorenanalyse berechnet. Dadurch konnten die Beziehungen zwischen den einzelnen Merkmalen im wesentlichen auf 3 Haupteinflussfaktoren zurückgeführt werden.

\section{Diskussion}

Jede quantitative Eigenschaft der Tiere ist durch die genetische Veranlagung und die Umweltwirkung determiniert. Dabei beeinflussen nicht nur gegenwärtige Verhältnisse die Ausbildung der Leistungseigenschaften, sondern es bestehen auch enge Verbindungen zu früheren Einwirkungen. Die vorliegenden Untersuchungen erstrecken sich üben mehrere Jahre. Die gute Fütterung und Haltung der Jungbullen während der Aufzucht in der Prüfstation bilden die Grundlage für eine vorzügliche Zuchtkondition. An den, unter einheitlichen Bedingungen aufgezogenen Bullen konnten Beziehungen zwischen Jugendwachstum und Spermaproduktion nachgewiesen werden. Die Korrelationswerte um 0,3 (Uusisalmi u.a. 1972) lassen erwarten, dass Bullen, 
Tabelle 2. Korrelationen zwischen der Aufzucht, der Spermaproduktion und der non-return Rate bei Zuchtbullen.

Non-return Rate der Besamungsjahre

alle ausgewer-

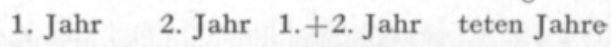

\begin{tabular}{|c|c|c|c|c|}
\hline \multicolumn{5}{|l|}{ Körperentwicklung } \\
\hline Lebendmasse am 150. Tag ....................... & -0.05 & 0.02 & $-0.32 *$ & $-0.36^{*}$ \\
\hline Lebendmasse am 180. Tag ....................... & -0.04 & 0.08 & $-0.32 *$ & $-0.33^{*}$ \\
\hline Lebendmasse am 210. Tag ........................ & -0.09 & -0.01 & $-0.38^{*}$ & $-0.37^{*}$ \\
\hline Lebendmasse am 240. Tag ....................... & -0.16 & 0.02 & $-0.39 * *$ & $-0.39 * *$ \\
\hline Lebendmasse am 270. Tag ....................... & -0.14 & 0.03 & $-0.38^{*}$ & $-0.36 *$ \\
\hline Lebendmasse;am 300. Tag ....................... & -0.15 & 0.06 & $-0.36^{*}$ & $-0.36^{*}$ \\
\hline Lebendmasse am 330. Tag ...................... & -0.12 & 0.02 & $-0.33^{*}$ & $-0.33^{*}$ \\
\hline Lebendmasse am 365 . Tag ....................... & -0.20 & 0.10 & -0.29 & $-0.31^{*}$ \\
\hline Tägliche Zunahme vom 121.-150. Tag ........ & -0.10 & -0.05 & $-0.32 *$ & $-0.36^{*}$ \\
\hline Tägliche Zunahme vom 60. -365 . Tag .......... & -0.21 & 0.12 & -0.26 & -0.25 \\
\hline Brustbreite am 180. Tag ......................... & -0.29 & 0.01 & $-0.49 * * *$ & $-0.49 * * *$ \\
\hline Hüftbreite am 180. Tag ........................... & -0.27 & -0.02 & $-0.48 * * *$ & $-0.50^{* * *}$ \\
\hline Beckenlänge am 180. Tag ....................... & $-0.37^{*}$ & -0.14 & $-0.54 * * *$ & $-0.57 * * *$ \\
\hline Brustumfang am 365. Tag ...................... & -0.21 & 0.16 & -0.26 & -0.26 \\
\hline Hüftbreite am 365 . Tag ................ & & 0.08 & $-0.37 *$ & $-0.37 *$ \\
\hline Beckenlänge am 365. Tag ........................ & . & -0.20 & $-0.57 * * *$ & $-0.49 * * *$ \\
\hline \multicolumn{5}{|l|}{ Spermaproduktion und Zuchtwert des Vaters } \\
\hline Spermamenge pro Monat ... & $0.32 *$ & -0.15 & 0.06 & 0.07 \\
\hline photometrische Zahl .............................. & $0.35^{*}$ & -0.14 & $0.38^{*}$ & $0.42 * *$ \\
\hline Zuchtwert für Fett $\%$ der Väter .................. & $-0.34^{*}$ & 0.23 & $-0.39 * *$ & $-0.45^{* *}$ \\
\hline \multicolumn{5}{|l|}{ Abhängigkeit zwischen den Besamungsjahren } \\
\hline non-return Rate 1. Jahr ................... & & -0.17 & $0.49 * * *$ & $0.56 * * *$ \\
\hline non-return Rate 2. Jahr ................. & & & 0.41 & 0.27 \\
\hline non-return Rate 1. 2. Jahr .................... & & & & $0.91 * * *$ \\
\hline
\end{tabular}

die eine bessere körperliche Entwicklung während der Aufzucht zeigen, zu einer vermehrten Spermaerzeugung fähig sind. $\mathrm{Zu}$ gleichen Resultaten kamen auch Czako und Veszely (1971) in ihren Versuchen.

Die Beziehungen zwischen der Lebendmasse der Tiere bei vorgegebenem Alter und den non-return Ergebnissen sind durch negative Verzeichen gekennzeichnet. Besonders deutlich tritt dies bei dem Gewicht am 240. Lebenstag in Erscheinung, $\mathrm{r}=-0,39 * *$. Eine gleiche Tendenz ist von Hladký, STraKovÁ and Straka (1970) nachgewiesen worden. Auch die tägliche Zunahme war negativ korreliert. Besonders auffällig sind die negativen Beziehungen zwischen den non-return Ergebnissen und den Körpermessungen am 180. Tag. Beckenlänge, Hüftbreite und Brustbreite waren mit $-0,57 * * *,-0,50 * * *$ und $-0,49^{* * *}$ sehr hoch signifikant. Auch am 365 . Lebenstag waren die Beckenlänge und die Hüftbreite, $r=-0,49 * * *$ und $r=-0,38^{*}$, gesichert korreliert, wie man aus Tabelle 2 ersehen kann. 
Werden die Bullen nach der Lebendmasse am 365. Tag entsprechend ihrer Stellung zum Mittelwert in zwei Klassen geordnet, so kann man mit dem $\chi^{2}$-Test gesicherte Unterschiede $(\alpha=1 \%)$ zwischen den leichteren und schwereren Bullen für die non-return Befunde nachweisen. Tabelle 3 zeigt

Tabelle 3. Vergleich der non-return Rate zwischen leichteren und schwereren Jungbullen.

\begin{tabular}{|c|c|c|c|}
\hline & \multicolumn{2}{|c|}{$\begin{array}{c}\text { Lebendmasse der untersuchten } \\
\text { Bullen am } 365 \text {. Tag }\end{array}$} & \multirow{2}{*}{$\begin{array}{l}\text { Anzahl } \\
\text { Rinder }\end{array}$} \\
\hline & 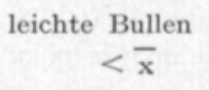 & $\begin{array}{c}\text { schwere Bullen } \\
>\bar{x}\end{array}$ & \\
\hline nicht nachbesamte Rinder ............. & 24928 & 18185 & 43113 \\
\hline nachbesamte Rinder .............................. & 7997 & 6179 & 14176 \\
\hline 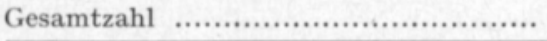 & 32925 & 24364 & 57289 \\
\hline non-return Rate in $\%$ & $75.71 \%$ & $74.64 \%$ & $75.26 \%$ \\
\hline
\end{tabular}

die einzelnen Werte. Bei den Spermaproduktionsmerkmalen gab es keine gesicherten Differenzen. An Hand weiterer Auswertungen der non-return Werte mit dem $\chi^{2}$-Test findet man zwischen den einzelnen Bullen sehr hoch gesicherte Unterschiede $(\alpha=0,1 \%)$. Es ist mit einer deutlichen genetischen Differenzierung der Bullen für dieses Merkmal zu rechnen. Das zeigen auch die, von Maijala (1967) für die finnischen Ayrshire errechneten Heritabilitätswerte der non-return Rate von 0,36 bis 0,47 . Die Korrelationen von $0,56 * * *$ zwischen den non-return Werten des 1 . Besamungsjahres und weiteren Besamungen deuten ebenfalls auf eine genetische Fixierung dieser Eigenschaft hin. Nach den non-return Ergebnissen der ersten beiden Besamungsjahre lässt sich eine gesicherte Aussage für die weiteren Besamungen machen, $r=$ $0,91 * * *$.

Auch der Einfluss der Milch- und Milchfettleistung der Eltern, als Zuchtwert registriert, auf die Befruchtungsfähigkeit des Bullenspermas wurde statistisch ausgewertet. Dabei zeigten sich negative, aber nicht gesicherteBeziehungen zwischen der Leistungsverbesserung der Eltern und den nonreturn Werten der Bullen. Nur für die Zuchtwerte der Fettprozente der Väter zu den non-return Raten ergab sich eine signifikante Korrelation -0,45**. Die Züchtung auf hohe Milch- und Fettleistung kann zur Verschlechterung der non-return Ergebnisse führen.

Berücksichtigen muss man auch, dass in den verschiedenen Jahreszeiten und den einzelnen Kalenderjahren gesicherte, unterschiedliche non-return Raten auftreten.

Bei der Vielzahl der untersuchten Eigenschaften erscheint es angemessen, die Faktorenanalyse zur Auswahl der wichtigsten Merkmalskomplexe heranzuziehen. Mit dieser Methode wurden drei wesentliche Faktoren gefunden. Sie sind hauptsächlich charakterisiert durch: 


\begin{tabular}{ll}
\hline Faktor & \multicolumn{1}{c}{ Merkmal } \\
\hline 1 & $\begin{array}{l}\text { Lebendmasse am 365. Tag } \\
\text { tägliche Zunahme vom 60. }-365 . ~ T a g \\
\text { Brustumfang am 365. Tag }\end{array}$ \\
\hline 2 & $\begin{array}{l}\text { Sprünge pro Absamtag } \\
\text { non-return Rate }\end{array}$ \\
\hline 3 & $\begin{array}{l}\text { Spermamenge pro Monat } \\
\text { Verwertbares Sperma pro Monat } \\
\text { Verwertbares Sperma pro Sprung } \\
\text { Verwertbares Sperma pro Sprungtag }\end{array}$ \\
\hline
\end{tabular}

Diese Faktoren geben einen Hinweis, welche Merkmale vorrangig beobachtet werden sollten bei den Zuchtbullen.

Die vorliegenden Untersuchungsergebnisse zeigen, dass es unbedingt notwendig ist, alle Eigenschaften der Zuchttiere komplex auszuwerten, da es sonst sehr schwierig ist, unerwünschte Entwicklungen rechtzeitig zu erkennen.

\section{Zusammenfassung}

Aufzucht, Fütterung und Haltung üben einen grossen Einfluss auf die Spermaproduktion und den Befruchtungserfolg der Zuchtbullen aus. Von 44 finnischen Ayrshirebullen der Besamungsstation Salpausselkä wurden die Aufzuchtergebnisse, die Spermaproduktion und die non-return Rate erfasst. Obwohl bei den mehrjährigen Untersuchunungen Zeiteinflüsse nicht ausgeschlossen werden können, wurden diese Unterlagen komplex ausgewertet. 57289 Erstbesamungen waren Grundlage für die Einschätzung des Befruchtungserfolges. Die erzeugte Spermamenge je Bulle und die Absamhäufigkeit hatten einen deutlichen Einfluss auf die Befruchtungsfähigkeit des Spermas.

Die einzelnen Bullen unterscheiden sich sowohl bei den Spermaproduktionsleistungen als auch in der non-return Rate gesichert. Die gefundenen Beziehungen zwischen den Aufzuchtleistungen und der Spermaproduktion waren positiv, zwischen den Aufzuchtleistungen und den non-return Ergebnissen waren vorwiegend negativ. Damit wird die Forderung einer komplexen Einschätzung aller Merkmale für Zuchttiere unterstichen.

\section{LITERATURNACHWEIS}

Czako, J. and Veszely, G. 1971. Effect of feeding of different intensity on growth and sexual ability of young replacer bulls. Acta Agron. Hung. Budapest, 20: 129-136.

Hladkx́, J., Straková, J. and Straka, P. 1970. Fertility of bulls in relation to their type and, above all, muscularity. Ná; Chov, Praha, 30:49-50.

HYLPS-Käsikirja. 1972. (HYLPS-Programmkatalog). Helsingin yliopiston laskentakeskus (Rechenzentrum der Universität Helsinki), Helsinki.

MaIJALA, K. 1967. Orsaker till variationen i semintjurarnas spermaproduktionsegenskaper och fruktsamhet. NJF-Kongressen, 27. -30. juni 1967, Køpenhamn, 158-159.

Uusisalmi, U., Kallio, M., Klautschek, G. \& VARo, M. 1972. Aufzucht und Spermaproduktion bei Jungbullen unter Stationsbedingungen in Finnland. J. Scient. Agric. Soc. Finl. 45: 217-226. 


\title{
Ayrshiresonnien kasvukoetulokset, spermantuotanto ja ei-uusintaprosentit Salpausselän keinosiemennysyhdistyksen alueella
}

\author{
G. KLAUTSChEK
}

Sektion Tierproduktion, Universität Rostock, DDR

U. Uusisalmi

Helsingin yliopisto, Kotieläinten jalostustieteen laitos

Keinosiemennyssonnien kasvatuksella, ruokinnalla ja hoidolla on merkitystä niiden siementuotantoon ja hedelmällisyyteen. Siksi analysoitiin Salpausselän keinosiemennysyhdistyksen alueella siemennyksiin käytetyn 44 ayrshiresonnin kasvukoeasematuloksia, spermantuotannon määrää ja laatua sekä siemennystuloksia. Sonnien ei-uusintaprosentit laskettiin yhteensä 57289 ensisiemennyksestä.

Ayrshiresonnit kasvatettiin vuoden ikään Humppilan kasvatusasemalla vuosina 1967-69. Eläimet punnittiin kasvukokeen aikana 60 päivän iästä lähtien 30 päivän välein. Lisäkasvut laskettiin. Lisäksi otettiin mittoja 180 päivăn ja 365 päivăn iässä. Sonnien siementuotannon määrää ja laatua mitattiin 12 kk:n iästä 24 kk:n ikään. Kultakin sonnilta vaadittiin vähintäin 7 kk:n tiedot spermasta. Sonnien ei-uusintaprosentit laskettiin vähintään kahdelta ensimmäiseltä siemennysvuodelta, eräiltä kuitenkin myös kolmannelta ja neljänneltä vuodelta. Ensisiemennysten luku vaihteli sonneittain 666:sta 3 509:ään. Havaintoarvoista laskettiin korrelaatiomatriisi, faktorianalyysi, t-testejä ja $\chi^{2}$-testejä.

Tuloksiksi saatiin mm.: Sonnien ei-uusintaprosentit vaihtelivat ensimmäisenä siemennysvuotena $62.3 \%$ :sta $90.0 \%$ :iin ja toisena vuotena $58.3 \%$ sta $89.2 \%$ :iin. Sonnien väliset erot todettiin merkitseviksi.

Aikaisemmassa tutkimuksessa Uusisalmi et al. (1973) totesivat, että kasvukokeessa hyvin menestyneet sonnit tuottivat myös hyvin spermaa. Tässä tutkimuksessa sen sijaan kasvukoetulokset korreloituvat negatiivisesti ei-uusintaprosenttien kanssa. Samoin ei-uusintaprosentit korreloituivat negatiivisesti $\left(\mathrm{r}=\boldsymbol{*}^{*} \boldsymbol{*}^{* *}\right) 180$ päivän ja 365 päivăn iässå otettuihin mittoihin.

Kun sonnit jaettiin kevyihin (paino vuoden iässä alle keskipainon) ja raskaihin (paino vuoden iässä yli keskipainon), todettiin kevyillä sonneilla saadut ei-uusinta-\%:t merkitsevästi suuremmiksi kuin raskailla.

Laskettiin sonnien ei-uusintaprosenttien korreloituminen niiden vanhempien absoluuttisiin ja suhteellisiin maito- ja rasvatuotoksiin ja todettiin yleensä negatiivista korreloitumista. Kuitenkin vain ei-uusinta-\%:n ja isien rasva-\%:n välinen korrelaatio oli tilastollisesti merkitsevä $\left(-0.45^{* *}\right)$. Tulosta voidaan pitää viitteenä sille, että tuotostason jalostuksellinen nostaminen saattaa johtaa alentuneeseen ei-uusinta-\%:iin.

Kasvukoetuloksista, sperman ominaisuuksista ja hedelmällisyyttä kuvaavista piirteistä saatiin faktorianalyysin avulla esiin kolme faktoria ja niitä kuvaavia tärkeimpiä piirteitä: 1 . f a kt o ri: 365 päivän paino ja lisäkasvu $60-365$ elopainon välillä; 2. f a k tori: hyppykertoja ottopäivää kohti ja ei-uusinta-\%; 3. f a k t o r i: sperman kokonaismäärä, ja hyväksytyn sperman määrä sekä ottopäiväă että hyppyä kohti.

Tutkimus osoittaa, että keinosiemennyssonnien spermantuotantokykyä ja ei-uusintaprosenttia on syytä arvostella yhdessä maidon- ja lihantuotantokyvyn kanssa, jotta voitaisiin ajoissa ehkäistä hedelmällisyysominaisuuksien mahdollinen epäsuotuisa kehitys. 